\title{
Circulations coloniales : \\ La place de l'Outre-mer dans la socialisation et les trajectoires professionnelles des policiers parisiens (1944-1962)
}

\author{
EMMANUEL BLANCHARD
}

De plus en plus, les historiens de l'administration intègrent la dimension impériale de la bureaucratie française ${ }^{1}$. Elle fut marquée à la fois par l'existence de corps spécifiques dédiés à la mise en valeur et à la régulation des territoires colonisés ${ }^{2}$, et par les allers-retours de fonctionnaires, de tous grades et tous statuts, entre la métropole et les possessions outre-mer ${ }^{3}$. Ce mouvement s'est accéléré dans les dernières années de la présence française en Algérie ${ }^{4}$, et avec les rapatriements successifs de personnels suite aux différentes décolonisations ${ }^{5}$. Ces circulations coloniales ne sont cependant réductibles ni à ce moment, ni aux seules carrières des fonctionnaires: elles peuvent intégrer d'autres dimensions liées par exemple aux caractéristiques biographiques et aux services militaires.

Ces transferts de personnel influèrent particulièrement sur la Sûreté nationale (SN). En 1956, elle fusionna avec la Sûreté algérienne et dut intégrer les fonctionnaires rapatriés des polices chérifiennes et tunisiennes. La Préfecture de police (PP) resta beaucoup plus à l'écart de ces mouvements ${ }^{6}$. L'exemple de la brigade nord-africaine (BNA) ${ }^{7}$ est pourtant la preuve

\footnotetext{
${ }^{1}$ M.-O. Baruch et V. Duclert, « Administrateurs et administrations sous la IV ${ }^{\mathrm{e}}$ République », Revue française d'administration publique, $\mathrm{n}^{\circ} 108,2003$, p. 501-520.

${ }^{2} C f$ l'étude pionnière de William Cohen sur les administrateurs coloniaux en Afrique. W. Cohen, Empereurs sans sceptre, histoire des administrateurs de la France d'outre-mer et de l'Etat colonial Paris, Berger-Levrault, 1971.

${ }^{3}$ Pour une approche générale et des études autour de cette problématique, T. Charbit et F. De Barros (dir.), « La colonie rapatriée », Politix, vol. 19, n 76, 2006.

${ }^{4}$ À partir de 1958, tous les élèves des grandes écoles (Polytechnique, ENA...) furent envoyés en stage en Algérie. M. MARIÉ, « La guerre, la colonie, la ville et les sciences sociales », Sociologie du travail, vol. XXXVII, $\mathrm{n}^{\circ} 2$ 2, 1995, p. 277-299.

${ }^{5}$ S. Laurens, «La noblesse d'Etat à l'épreuve de "l'Algérie" et de l'après 1962. Contribution à l'histoire d'une “cohorte algérienne" sans communauté de destins », Politix, vol. 19, n 76, 2006, p. 75-96.

${ }^{6}$ La PP était une administration locale et les fonctionnaires de police d'Indochine ou du Maghreb qui souhaitaient l'intégrer devaient passer un concours. Des recrutements contractuels permettaient cependant de déroger à cette règle. Archives de la Préfecture de police (APP) HA 1.

${ }^{7}$ De 1926 à 1945, un service d'assistance aux indigènes nord-africains (SAINA) a fonctionné sous l'égide de la PP. Basé rue Lecomte, dans le $17^{\mathrm{e}}$ arrondissement, il incluait la BNA. Elle était notamment chargée de la surveillance politique des Algériens. Elle pouvait s'appuyer sur des services d'assistance qui lui étaient entièrement subordonnés. Le SAINA et la BNA furent dissous à la Libération. E. BLANCHARD, «La dissolution des Brigades nord-africaines de la Préfecture de police : la fin d'une police d'exceptions pour les Algériens de
} 
flagrante, que la PP avait, par le passé, su constituer, selon des modalités dérogatoires, un groupe de policiers caractérisés par leurs expériences et leurs connaissances des colonies ou des colonisés. Ce « capital colonial ${ }^{8}$ » pouvait avoir été accumulé de différentes manières : par le fait d'avoir été régi par le statut de colonisé tout en ayant atténué ce stigmate originel par la naturalisation ${ }^{9}$; en ayant vécu en territoire colonisé, particulièrement en y étant né ; en ayant travaillé avec, et surtout, commandé des "indigènes", particulièrement dans le cadre de l'armée.

Cette importance de l'expérience coloniale caractéristique des anciens de la BNA, les distingue du reste des personnels de la PP, en particulier des gardiens de la paix, est pourtant loin de les isoler totalement. Tout au long de l'après-guerre, les préfets successifs étaient ainsi particulièrement insérés dans des réseaux coloniaux, qu'ils soient professionnels ou d'affinités politiques.

\section{Des préfets de police intégrés à l'espace colonial}

Il n'existait pas à proprement parler de parti ou association politique structurant la défense des intérêts des partisans de l'empire puis de l'Union française. Les colons et autres militants actifs de la présence française outre-mer avaient cependant constitué un certain nombre d'organismes chargés de défendre l'œuvre de la colonisation et bénéficiaient de relais politiques au parlement. Bien qu'il soit entré en déclin après la Seconde Guerre mondiale, ce « parti colonial ${ }^{10}{ }$ ) continuait d'avoir une certaine influence. Les anticolonialistes étaient eux totalement absents de la scène parlementaire et ministérielle ${ }^{11}$. Au sein du champ politique s'affrontaient cependant plusieurs visions de l'Union française. Celle d'un Edouard Depreux, ancien avocat de Messali Hadj dans les années $1930^{12}$, futur fondateur du PSU, et ministre de l'Intérieur au moment de la négociation de l'Algérie française, n'avait ainsi rien à voir avec celle de René Mayer - député radical de Constantine, (dé)tricoteur de majorités parlementaires et de cabinets ministériels, et strict défenseur des intérêts des colons algériens.

Paris $»$, Bulletin de l'IHTP, $\mathrm{n}^{\circ} 83,2004$, p. 70-82.

${ }^{8}$ C. HMED, "“Tenir ses hommes". La gestion des étrangers "isolés" dans les foyers Sonacotra après la guerre d'Algérie », Politix, vol. 19, n 76, 2006, p. 30.

${ }^{9}$ À une exception près, tous les colonisés d'Algérie exerçant rue Lecomte avaient été "naturalisés", que ce soit avant ou après leur entrée au SAINA. APP dossier d'épuration d'Ali Ouarab.

${ }^{10}$ C-R. Ageron, France coloniale ou parti colonial ?, Paris, PUF, 1978.

${ }^{11}$ Jusqu'en 1947, le PCF est un ardent défenseur de l'Algérie française qu'il s'agit notamment de soustraire aux appétits américains. Son soutien à l'indépendance n'interviendra que très tardivement puisqu'en mars 1956 il vota les pouvoirs spéciaux.

${ }^{12}$ E. Depreux, Souvenirs d'un militant. De la social-démocratie au socialisme, un demi-siècle de lutte, Paris, Fayard, 1972, p. 38. 
Après l'adoption du statut de l'Algérie en septembre 1947, jusqu'au début des décolonisations, la question du devenir de l'Union française n'a plus été en tête de l'agenda politique. Elle était cependant prise en compte dans les tractations préalables au choix du titulaire du maroquin de l'Intérieur : après Adrien Tixier (1945-1946) et Édouard Depreux (1946-1947), dont les conceptions de l'Union heurtaient les intérêts des colons ${ }^{13}$, ce ministère fut par la suite occupé par des hommes proches des groupes d'intérêts coloniaux. Si Jules Moch (1947-1949) s'est présenté a posteriori comme un partisan de solutions négociées en Algérie et en Indochine, il était surtout peu intéressé par les questions coloniales ${ }^{14}$. Du fait de son anticommunisme, il était enclin à ne pas heurter les intérêts des élus d'outre-mer et à ne surtout rien entreprendre qui pût paraître une concessions au PCF. À partir de 1950, le ministère tomba durablement dans l'escarcelle de l'orbite radicale (parti radical, radicalsocialiste, RGR, UDSR) aux mains d'hommes (Charles Brune, Léon Martineau-Déplat) nommés pour leurs proximités avec les élus les plus anticommunistes et non moins ardents défenseurs du statu quo dans l'Union française. La question coloniale n'était alors pas centrale, elle le devint avec les premières décolonisations. À partir de 1954, elle a été un puissant facteur d'instabilité ministérielle et de déstabilisation politique, entravant durablement les carrières d'hommes tel Mendès France, tombé notamment pour s'être opposé $\mathrm{au}$ « lobby colonial ${ }^{15}$ » et avoir remis en cause le modus vivendi selon lequel la présidence du Conseil ne pouvait pas heurter de front les intérêts du parti colonial. René Mayer fut ainsi en première ligne dans la coalition qui fit chuter Pierre Mendès France en février $1955^{16}$. Après la parenthèse ambiguë du passage de François Mitterrand place Beauvau ${ }^{17}$, le portefeuille de l'Intérieur fut à nouveau arrimé en des mains (Maurice Bourgès-Maunoury, Jean GilbertJules) dont on pouvait être sûr qu'elles ne trembleraient pas en cas de nécessaires répressions de menées indépendantistes.

L'exemple de la chute de Mendès France montre de quelle manière questions de police et intérêts coloniaux étaient étroitement liés. François Mitterrand, alors ministre de l'Intérieur de Mendès-France, l'interpréta comme une sanction contre sa volonté d'intégrer la police d'Algérie à la Sûreté nationale afin de mieux lutter contre les violences et tortures pratiquées outre-Méditerranée : «Il est certain que la fusion des polices métropolitaine et algérienne et

\footnotetext{
${ }^{13}$ Les polémiques au cours du voyage de Depreux en Algérie en avril 1947 en attestent. AN 456 AP/3 (fonds Depreux).

${ }^{14}$ J. Moch, Une si longue vie, Paris, Robert Laffont, 1976.

${ }^{15}$ E. Duhamel, Histoire politique de la IV République, Paris, La Découverte, 2000, p. 81.

${ }^{16}$ E. Roussel, Pierre Mendès France, Paris, Gallimard, 2007, p. 378-386.

${ }^{17}$ François Mitterrand est resté dans l'histoire comme le ministre de l'Intérieur ayant déclaré devant l'Assemblée nationale, au lendemain de la Toussaint rouge, «L'Algérie c'est la France ». Il était pourtant honni par le grand colonat algérien. Voir infra.
} 
que la mutation des chefs de la police algérienne constituèrent l'une des causes principales, sinon la première, de l'hostilité bientôt irréductible que nous montrèrent les maîtres du jeu algérois...La police constituait l'un des atouts maîtres du lobby algérien : la menace, le chantage, la violence lui permettaient de tenir les fonctionnaires, la presse, les hommes politiques. Nous avons donc frappé un système détestable sur un point sensible. Vous connaissez la suite ${ }^{18}$. »

La fusion des polices fut envisagée pour gommer les spécificités d'une Sûreté algérienne qui n'avait pas rompu avec certaines pratiques de la conquête ${ }^{19}$, y compris la torture, dont la fréquence a été dénoncée jusque dans des rapports du directeur de la Sûreté nationale ${ }^{20}$. Elle était aussi marquée de longue date par la collusion avec les grands colons, à la fois détenteurs du pouvoir économique, de la presse et des postes électifs ${ }^{21}$. Même s'ils étaient mois sujets à ces pression que les responsables de la police en Algérie, ce sont ces rapports des préfets de police successifs avec le monde colonial que nous souhaiterions maintenant questionner.

Louis Lépine est resté dans les mémoires comme l'archétype des préfets de police. La longévité de sa charge (1893-1897; 1899-1913), sa médiatisation, et ses innovations en matière de maintien de l'ordre notamment ${ }^{22}$, ont marqué l'île de la Cité où il a laissé une empreinte durable. Pour notre sujet, sa carrière est remarquable du fait qu'entre ses deux passages à la Préfecture de police, il fut nommé gouverneur général de l'Algérie (1897) pour ramener l'ordre troublé par « la crise antijuive et (...) autonomiste ${ }^{23} »$. Malgré ses états de service, il ne « réussit [qu'] à provoquer l'unanimité contre lui ${ }^{24} »$. Il ne resta que quelques mois, échoua à ramener le calme et empêcher l'élection de Drumont, et fut rappelé à Paris

\footnotetext{
${ }^{18}$ Lettre manuscrite de François Mitterrand à Pierre Mendès France, 31 août 1959, Institut Pierre Mendès France, citée par : J-P. Peyroulou, «Rétablir et maintenir l'ordre colonial : la police française et les Algériens en Algérie française de 1945 à 1962 » in M. HARBi et B. Stora (dir), La guerre d'Algérie. 1954-2004, la fin de l'amnésie, Paris, Robert Laffont, 2004, p. 118.

${ }^{19}$ De fait cette fusion des polices a surtout permis d'envoyer plus facilement des compagnies de CRS en Algérie. Elle permit aussi d'y faire transiter les policiers rapatriés du Maroc et de Tunisie qui durent y faire un stage de 6 mois avant que de nouveaux postes, souvent en Algérie, ne leur soient proposés. APP HA 1.

${ }^{20}$ " La pratique de la torture était aussi ancienne que la police en Algérie » (Peyroulou J-P., op. cit. , p. 117). Jean Mairey, directeur de la Sûreté nationale nommé par François Mitterrand, en décrit les mécanismes dans deux rapports de mars et décembre 1955. P. Vidal-Naquet, La raison d'Etat. Textes publiés par le comité Audin, Paris, Maspéro, 1962.

${ }^{21}$ Henri Borgeaud, propriétaire terrien et industriel, conseiller de la République RGR (1946-1958) possédait aussi La dépêche quotidienne dans laquelle Laurent Schiaffino, armateur, sénateur et président de la chambre économique d'Alger avait des parts. Georges Blachette, « roi de l'alfa », député républicain indépendant (19511955), était propriétaire du Journal d'Alger. Borgeaud avait également la haute main sur les nominations administratives, notamment dans la police. Avant d'être nommé puis installé à Alger, Jacques Léonard évoque, dans ses carnets, plusieurs entretiens avec René Mayer. Celui-ci relayait notamment les préventions de Borgeaud à l'égard d'un des adjoints du préfet de police, accusé de proximité avec la SFIO. Agendas Léonard, 5 avril 1951, Centre d'histoire de Science-Po.

${ }^{22}$ J-M. BerLière, Le préfet Lépine. Vers la naissance de la police moderne, Paris, Denoël, 1993.

${ }^{23}$ C-R. Ageron, Histoire de l'Algérie contemporaine. De l'insurrection de 1871 au déclenchement de la guerre de libération (1954), Paris, PUF, 1979, p. 62.

${ }^{24}$ J.-M.BERLIÈRE, op.cit. , p. 87.
} 
pour répondre aux revendications et apaiser la colère des « Algérianistes ${ }^{25} »$. Cette expérience est exemplaire en ce qu'elle préfigure les trajectoires professionnelles de plusieurs des préfets de l'après-guerre. Ainsi le gouvernement général de l'Algérie fut périodiquement remis entre les mains de spécialistes de maintien de l'ordre, notamment quand il s'agissait de donner des gages à une population européenne inquiète de l'agitation des Musulmans. A contrario, l'expérience coloniale pouvait être envisagée comme une compétence adéquate pour maintenir l'ordre à Paris, capitale dont les soulèvements populaires périodiques avaient longtemps inquiété le pouvoir.

La carrière de Maurice Papon fut emblématique de ces allers-retours entre la métropole et les colonies. Placée sous les auspices d'une compétence reconnue en matière de maintien de 1'ordre ${ }^{26}$, elle le fut aussi sous celle d'un “patron” René Mayer, et d'un premier protecteur Maurice Sabatier ${ }^{27}$. Ils ont pesé de tout leur poids pour qu'il fut nommé au gré des impératifs et des intérêts du parti colonial ${ }^{28}$.

Après-guerre, Papon fut nommé à la préfecture de Constantine. Il entra en poste juste après les événements de Sétif (8 mai 1945), assista à la sanglante répression des insurgés et de la population civile qui s'en suivit. Après un interlude comme préfet de Corse, il revint dans l'est algérien. Préfet, il était en poste lors du démantèlement de l'organisation spéciale du MTLD (printemps 1950). Cette vaste opération de police s'est accompagnée de tortures quasi-systématique sur les 363 suspects interpellés. C'est à l'issue de cette mission qu'il effectua son premier séjour à la PP (1951-1954), en tant que secrétaire général du préfet Baylot. Celui-ci, obsédé par le danger communiste, voyait dans le MTLD une simple succursale du PCF. Il mena une politique très dure à l'égard des Algériens, multiplia les rafles, pratiqua sur une grande échelle l'internement préventif avant les manifestations nationalistes. Surtout, il réprima dans le sang le cortège du 14 juillet $1953^{29}$. Après que Baylot

\footnotetext{
${ }^{25}$ C.-R.Ageron , op.cit. , p. 64.

${ }^{26}$ Aujourd'hui encore certains anciens de la PP lui reconnaissent cette compétence et regrettent qu'il ait dû quitter la PP en 1967 et n'ait pas été à leur tête en mai 1968. Entretien avec M. Jean* (brigadier de compagnie de district), Paris, 4 janvier 2007.

${ }^{27}$ Maurice Sabatier, né en Algérie, membre du part radical, avait été un des premiers protecteur du jeune Papon. Secrétaire général au ministère de l'Intérieur (1941-1942), il avait sous sa charge les affaires algériennes et prit part à l'application des mesures antisémites contre les Juifs d'Algérie. Il initia Papon aux subtilités de la politique algérienne et lui fit découvrir l'Afrique du Nord. Préfet régional de l'Aquitaine (1942-1944), c'est sous son autorité que Maurice Papon continua sa carrière à Bordeaux puis en Algérie où les plus compromis des hommes de Sabatier impliqués dans les persécutions antisémites de Bordeaux trouvèrent avec lui refuge en 1945. Inculpé de crime contre l'humanité (1987), Maurice Sabatier est décédé en avril 1989 avant de comparaître.

${ }^{28}$ J. House et N. MacMaster, Paris 1961. Algerians, State Terror, and Memory, Oxford, Oxford university press, 2006, p. 33-50.

${ }^{29}$ Le 14 juillet 1953, placés en queue de cortège, les Algériens du MTLD qui arrivaient place de la Nation voulurent continuer de défiler après l'ordre dispersion donné par la police. Celle-ci chargea, ouvrit le feu, et tua sept manifestants dont six Algériens.
} 
a été démis de ses fonctions par Mendès France suite à la tortueuse affaire des fuites (juillet $1954)^{30}$, la réputation d'homme à poigne et l'expérience coloniale de Papon ne restèrent pas longtemps inemployées. Quelques jours après avoir quitté la PP, il était nommé à la résidence générale du Maroc en tant que secrétaire général, où avec le résident Francis Lacoste ils furent les hommes de « la coterie des ultras-coloniaux ${ }^{31}$ ». Ils accentuèrent une " évolution policière du régime " symbolisée avant leur arrivée par la déposition du Sultan en août 1953. Ils laissèrent le champ libre aux «contre-terroristes assassin[ant] impunément ${ }^{32}$ ». Francis Lacoste fut rappelé en juin 1955 par Edgard Faure qui voulait rompre l'engrenage de la violence au Maroc. Papon se trouva alors quelques mois en disgrâce et sans affectation, avant que son profil ne fut à nouveau en phase avec le contexte politique. Quelques semaines après le vote des Pouvoirs spéciaux, il était nommé IGAME (Inspecteur général de l'administration en mission extraordinaire) de Constantine (1956-1958) où il se fit propagandiste et organisateur de l'action psychologique et de la « guerre contre-révolutionnaire ». C'est au vu de ses états de service qu'il fut rappelé à la tête d'une Préfecture de police ébranlée par la guerre d'Algérie et par la manifestation des gardiens de la paix du 13 mars $1958^{33}$.

Les affectations de Papon à la PP succédèrent toutes les deux à des périodes où il était en fonction en Afrique du Nord, en charge notamment de la répression des mouvements nationalistes. Même si les modalités et l'intensité de ces phases de répression policières - puis militaires entre 1956 et 1958 - diffèrent selon les périodes, certaines continuités existent. Neil MacMaster met ainsi en évidence l'importance du laboratoire marocain en matière d'action psychologique, tant pour la période où Papon fut en fonction comme IGAME qu'à la PP après 1958. Papon y rapatria ainsi des officiers des affaires indigènes marocains qui l'avaient déjà suivis de Rabat à Constantine ${ }^{34}$.

Si les allers-retours des titulaires de la charge de préfet de police entre Paris et les colonies n'ont rien d'exceptionnel (cf. infra), le profil de Papon, inséré dans les réseaux du parti colonial, préfet ne fixant aucune limite à la violence des opérations de maintien de l'ordre, chantre de la «guerre contre-révolutionnaire », est par contre très spécifique. Il diffère beaucoup de celui d'autres préfets de police au capital colonial presque aussi affirmé.

\footnotetext{
${ }^{30}$ Sur les accusations portées par le réseau Dides de la Préfecture de police, contre certains ministres accusés de trahison au profit du PCF et donc de l'URSS : P. Marcus, La République trahie. L'affaire des fuites (1954), Biarritz, Atlantica, 1999.

${ }^{31}$ D. Rivet, Le Maghreb à l'épreuve de la colonisation, Paris, Hachette, 2002, p. 387.

${ }^{32} \mathrm{Ibid}$, p. 391, 397.

${ }^{33}$ Une manifestation revendicative à l'appel des syndicats de gardiens déborda de la cour de la PP. Des cortèges se dirigèrent vers l'Assemblée nationale devant laquelle cette foule houleuse, haranguée par des députés poujadistes, dont Jean Dides et Jean-Marie Le Pen, stationna plusieurs heures. Le préfet Lahilonne démissionna le lendemain.

${ }^{34}$. J. House et N. MACMASTER , op. cit. , p. 46.
} 
Ainsi, André-Louis Dubois, issu d'une famille de colons installés depuis trois générations à Bône, a, quelques années avant Papon, lui aussi commencé sa carrière sous les auspices d'un des élus radicaux les plus en pointe dans la défense du projet colonial. Membre de plusieurs cabinets d'Albert Sarraut (1932-1938), il fut par la suite nommé directeur à la Sûreté nationale (1938-1940). Ce «fonctionnaire indigne ${ }^{35} »$ fut contraint d'abandonner ce poste après que la loi du 17 juillet 1940 sur le relèvement des fonctions fut entrée en application. À la Libération, il retourna à la SN qu'il dut à nouveau quitter, cette fois devant une offensive du nouveau ministre, Édouard Depreux, qui choisit de placer des hommes du parti socialiste ${ }^{36}$. C'est peu après que la préfecture d'Alger lui fut proposée ${ }^{37}$.

Or, la politique du gouverneur général de l'Algérie, le général Chataigneau, ne lui convenait pas : la qualifiant 25 ans après les faits de «mi-chèvre mi-chou », il considérait qu'elle ne pouvait conduire qu'au « massacre ${ }^{38} »$. Au contraire des représentants des colons qui obtinrent en 1948 le départ d'Yves Chataigneau, considéré comme trop proche des Français musulmans, Dubois trouvait que sa politique manquait d'audace. Il déclare dans ses mémoires avoir été partisan d'octroyer la pleine citoyenneté à tous les Algériens et d'en assumer l'ensemble des conséquences. Quelle que soit l'importance, dans ces propos autobiographiques, des reconstructions et des regrets des occasions manquées dans un processus de décolonisation qui aurait pu mener à un «commonwealth français ${ }^{39}$ », André Dubois était, sous la IV ${ }^{\mathrm{e}}$ république, perçu comme un libéral en matière coloniale. La suite de sa carrière en atteste : choisi en juillet 1954, par Mendès France, pour remplacer Baylot et faire évoluer un certain nombre de pratiques à la PP, ensuite pressenti pour remplacer Léonard - trop bienveillant avec les "ultras" - au gouvernement général de l'Algérie ${ }^{40}$, il quitta l'île de la Cité pour devenir résident général à Rabat. Il y rétablit le Sultan sur le trône et prépara «l'indépendance dans l'interdépendance ${ }^{41}$ " avant de devenir pour quelques mois le premier ambassadeur de France au Maroc (1955-1956).

Ainsi, Maurice Papon et André Dubois se livrèrent à une sorte de chassé-croisé. D'abord à la $\mathrm{PP}$, puis à Rabat, le second fut appelé à remplacer le premier, quand les conséquences néfastes de la répression et de la surveillance à outrance initiées par le premier faisaient par

\footnotetext{
${ }^{35}$ Note non datée de la Sûreté générale adressée au maréchal Pétain citée in M.-O. BARuch, Servir l'État français. L'administration en France de 1940 à 1944, Paris, Fayard, 1997, p. \$\$.

${ }^{36}$ A.-L. Dubois, À travers trois Républiques. Sous le signe de l'amitié, Paris, Plon, 1972, p. 236.

${ }^{37}$ Ibid, p. 237-238.

${ }^{38}$ Ibid.

${ }^{39}$ A-L Dubois et P. Sergent, Le malentendu algérien : 12 ans après, Paris, Fayard, 1974.

${ }^{40}$ Finalement, au lieu de Dubois, suggéré par Mitterrand, Pierre Mendès France choisit Jacques Soustelle qui était aussi renommé libéral en la matière, réputation qu'il n'allait pas tarder à faire mentir.

${ }^{41}$ Selon « la formule trop habile pour être efficace » d'Edgard Faure. E. DuHAmEL, op. cit. , p. 73.
} 
trop ressentir leurs conséquences négatives. Ces exemples montrent que derrière des trajectoires professionnelles proches et une même importance de la composante coloniale dans leurs carrières, se cachent des visions politiques et surtout une manière d'envisager la police et le maintien de l'ordre fort différentes ${ }^{42}$.

Des trajectoires et une expérience coloniale proches ne suffisent donc pas à définir un ethos professionnel commun. Si les pratiques et les politiques des préfets de police varient considérablement, il ne semble pas que ce soit dans le passé colonial qu'il faille aller chercher les clés de leur influence personnelle sur le fonctionnement de la PP. La rapide analyse des carrières des préfets de police qui se sont succédés sous la $\mathrm{IV}^{\mathrm{e}}$ République $^{43}$ permet cependant de mettre en évidence que le monde colonial faisait partie intégrante de leur champ professionnel.

Sur les sept préfets nommés entre 1944 et 1958, cinq ont eu d'importantes responsabilités en Algérie et au Maroc. Ces trajectoires professionnelles confirment les observations faites pour d'autres administrateurs: l'Outre-mer faisait partie intégrante des déroulés de carrière des hauts fonctionnaires et l'Algérie était même perçue comme un prolongement de la métropole dont la gestion bureaucratique ne se différenciait guère de celle de la rive nord de la Méditerranée ${ }^{44}$. L'Outre-mer dont le prestige était au zénith, à la Libération, pour les candidats aux métiers de la fonction publique, ne proposait donc pas seulement des carrières spécifique. Elle offrait aussi des ressources, des débouchés importants pour ceux qui étaient arrivés au terme d'une trajectoire réussie dans l'administration métropolitaine. Ainsi, pour les membres du corps préfectoral -6 des 7 préfets de police de la période ${ }^{45}$ - qui ont fait toute leur carrière en métropole, la Préfecture de police est un apogée qui vient souvent conclure des passages réussis par les préfectures les plus importantes. À l'issue de leur mandat, quand ils ne sont pas démis de leurs fonctions et tombés en disgrâce politique (Baylot, Lahilonne), les préfets de police n'ont donc plus rien à attendre d'une affectation dans le même corps. Ils doivent rechercher de nouveaux horizons : «Je ne m'accroche nullement à la PP et accepterais très certainement un poste tel que l'Algérie ou le Maroc, s'il devait devenir vacant, ce dont il n'est aucunement question pour l'instant ${ }^{46} . »$ "Personnellement je ne tiens

\footnotetext{
${ }^{42}$ A-L.Dubois , op. cit. ; M. PAPON, Les chevaux du pouvoir. Le Préfet de police du général de Gaulle ouvre ses dossiers, 1958-1967, Paris, Plon, 1987.

${ }^{43}$ Le premier préfet de police nommé sous la V ${ }^{\mathrm{e}}$ République est Maurice Grimaud, successeur de Papon en 1967.

${ }^{44}$ J-C. FREDENUCCI, « La brousse coloniale ou l'anti-bureau », Revue française d'administration publique, $\mathrm{n}^{\circ} 108$, 2003, p. 603-616.

${ }^{45}$ Seul Luizet a un parcours totalement atypique. Diplômé de Saint-Cyr, militaire outre-mer, préfet de la Corse libérée, il a intégré la PP à la faveur de son engagement dans la Résistance.

${ }^{46}$ Agendas Léonard, 7 février 1949.
} 
aucunement à étendre mes responsabilités et mes attributions mais (...) je ne puis accepter d'être coiffé par un fonctionnaire de rang inférieur au mien ${ }^{47}$.

Les préfets de police qui ne se virent pas offrir de prestigieuses fonctions Outre-mer cessèrent d'ailleurs leur carrière administrative pour se reconvertir en politique (Baylot, élu député sous l'étiquette «Indépendants paysans », 1958-1962) ou dans les affaires (Genebrier, Lahilonne). Ces reconversions ne sont d'ailleurs pas sans connexion avec l'empire colonial ${ }^{48}$.

Les préfets de police de Paris apparaissent ainsi, pour des raisons diverses - biographiques, intégration à des réseaux politiques - mais aussi communes - postes prestigieux à pourvoir fortement intégrés à un monde colonial auxquels a minima des intérêts stratégiques de déroulés de carrières les relient. Cet espace professionnel ultramarin n'était pas sans influer sur l'attachement des hauts fonctionnaires à l'Union française. Ces caractéristiques préfectorales étaient cependant loin d'être communes à l'ensemble du personnel de l'île de la Cité.

\section{Le vécu colonial des gardiens de la paix parisiens}

Les archives de la PP ne conservent pas les dossiers administratifs des gardiens de la paix et une petite partie seulement de ceux des autres personnels qui rend difficile toute étude prosopograhique. Nous avons pris le parti de contourner cette difficulté de deux façons : par le traitement des dossiers administratifs des «victimes du devoir» de 1945 à $1962^{49}$ et l'utilisation des récits de vie de policiers. Du premier échantillon, nous n'avons conservé que les personnels ayant débuté au grade de gardien de la paix soit 72 dossiers sur 76 . Le second, 21 récits de vie de policiers parisiens ayant été gardiens de la paix au cours de la période 1944-1962, fait l'objet d'une analyse moins systématique, du fait de son étroitesse, mais permet de contrôler le premier sur certains points ${ }^{50}$.

Cet échantillon est loin d'être homogène puisque le nombre de policiers «victimes du devoir » est beaucoup plus important aux deux bornes chronologiques : le chiffre important pour l'année 1945 s'explique notamment par le nombre de policiers morts en déportation (15)

\footnotetext{
${ }^{47} \mathrm{Ibid}, 6$ novembre 1950.

${ }^{48}$ Roger Genebrier dirige des mines d'Algérie. Jean Baylot qui en tant que préfet de police avait été fortement soutenu par le parti colonial, tisse au travers de ses responsabilités maçonniques de solide réseaux "Françafricains". F.-X. Verschave, Noir Chirac, Paris, Les Arènes, 2002, p. 73-75.

${ }^{49}$ L'ensemble du fonds « victimes du devoir » inventorié sur les listes des APP, soit 76 dossiers pour les années 1945-1962, a fait l'objet d'un dépouillement systématique.

${ }^{50}$ Sur un total de 94 entretiens recueillis dans le cadre du programme de l'IHESI-INHES, nous avons retenu un corpus de 82 récits de vie duquel ont été sélectionnés pour cette étude ceux de 21 gardiens de la paix à la PP. Ces récits de vie sont consultables à la BNF.
} 
et les suites des combats de la Libération (3); de 1958 à 1962 la quasi-totalité des victimes du devoir ont été tués en service par des membres du FLN (27 sur 32). Les policiers morts en déportation sont pour la plupart représentants de l'infime minorité entrée dans une résistance active avant l'année 1944, mais aucune autre caractéristique spécifique ne semble les distinguer des autres gardiens. Par ailleurs, seule une minorité des policiers tués par le FLN était spécialisée dans la répression des nationalistes algériens, tâche pour laquelle ont été mobilisés à un moment ou à un autre des années 1958-1962, la majorité des gardiens. La véritable spécificité de ce groupe tient à la durée (12 ans en moyenne) et au déroulement de carrières prématurément interrompues. Cet échantillon intègre aussi très mal les recrues des années de la guerre d'Algérie, qui sont peu à peu venues renouveler des effectifs restés très stables après les renouvellements intervenus sous Vichy et à la Libération. La date moyenne d'entrée à la PP est 1940, et seuls six gardiens ont été recrutés après 1954. Ces derniers font partie des cohortes dont le recrutement a été directement ou indirectement lié à la guerre d'Algérie. Au-delà des seuls (r)appelés qui bénéficiaient de certaines facilités pour intégrer la police, des CRS et gendarmes mobiles démissionnèrent de leurs fonctions pour éviter de retourner en Algérie et passèrent les concours de la $\mathrm{PP}^{51}$, des policiers rapatriés firent de même. Les policiers entrés en fonction dans les années 1970 sont nombreux à rappeler qu'ils étaient massivement encadrés par des anciens d'Algérie ${ }^{52}$.

A l'instar de leurs collègues, ces gardiens de la paix apparaissent donc comme massivement issus des milieux ouvriers, en particulier de région parisienne ${ }^{53}$. Ils sont entrés à la PP après avoir travaillé plusieurs années et satisfait à leurs obligations militaires ${ }^{54}$. Jusqu'au milieu des années 1950, le métier de policier était alors un moyen d'échapper à la précarité et l'entrée à la PP était très souvent liée à des projets familiaux (mariage, naissance).

Outre ces proximités sociales entre les recrues de la PP et les ouvriers parisiens, on peut aussi pointer une "génération 1939-1945" tant est importante la part des policiers de l'après-guerre entrés, au cours, ou à la suite, du second conflit mondial ${ }^{55}$. Nous pouvons aussi relever qu'aucun des policiers de notre échantillon n'est né hors de métropole et qu'environ un tiers a fait le choix de prolonger ses obligations militaires au-delà du seul service obligatoire.

\footnotetext{
${ }^{51}$ Témoignage de M. Jean*, Paris, 4 janvier 2007.

${ }^{52}$ Voir, par exemple, les récits de vie de Claude Donadille, Patrick Hazo et Francis Laheurte.

${ }^{53}$ On retrouve cette même proportion, d'environ $50 \%$ des gardiens nés en région parisienne, dans le corpus des récits de vie (11 sur 21).

${ }^{54}$ Une part relativement importante des gardiens ( $1 / 6^{\mathrm{e}} \mathrm{du}$ corpus de « victimes du devoir »; $1 / 4$ du corpus récits de vie) entrés pendant la guerre ou à la Libération, n'a cependant jamais fait de service militaire, sans compter ceux qui l'ont effectué au sein des pompiers de Paris.

${ }^{55} 8 / 21$ pour le corpus récits de vie, 28/72 pour le corpus victimes du devoir. Au cours de la période étudiée, il est possible de retenir comme ordre de grandeur, que le pourcentage des gardiens recrutés entre 1939 et 1945 a décliné du fait des nouveaux recrutements d'un peu moins de $40 \%$ à environ un tiers.
} 
Sur 15 gardiens (soit $20 \%$ de notre échantillon) ayant accumulé une expérience coloniale, seul un l'a fait hors du cadre de l'institution militaire. Tous les autres ont servi dans l'empire français comme appelés ou engagés. Sur ces 14 soldats coloniaux au moins 11 sont passés par l'Afrique du Nord, la plupart pour des durées n'excédant pas une à deux années. Au moins deux ont participé aux campagnes du Maroc des années 1920, un à la guerre d'Indochine et un à la guerre d'Algérie. La place de ces deux derniers conflits dans la trajectoire biographique et professionnelles des policiers parisiens des années 1950-1960 est cependant largement sous-estimée par la structure même de notre échantillon. Peu de données sont disponibles afin d'évaluer correctement le nombre de policiers étant passés par les champs de batailles d'Indochine et d'Algérie, mais ils constituaient une part non négligeable des personnels de la PP à la fin de la guerre d'Algérie. Un rapport de décembre 1961 notait ainsi que 490 membres des services actifs de la PP avaient servi en Indochine ${ }^{56}$, et des facilités avaient été mises en place pour favoriser le recrutement des anciens combattants d'Algérie ${ }^{57}$. Si l'on ajoute le corpus de récits de vie à celui de victimes du devoir, ce paramètre de la participation aux guerres coloniales est ainsi un peu mieux pris en compte puisque sur trois gardiens recrutés après 1956, deux ont effectué leur service militaire en Algérie. Plus généralement, un tiers des gardiens ( 7 sur 21) ont accumulé une expérience coloniale soit un pourcentage significativement plus élevé que celui des « victimes du devoir» $(20 \%)$. Cette différence est due au fait que la date moyenne d'entrée dans la police est plus tardive pour ces gardiens que pour ceux du corpus récit de vie. Elle accrédite donc l'hypothèse d'une augmentation de l'expérience coloniale des gardiens à la fin de la période étudiée, en raison de leur participation aux guerres de décolonisation.

Deux éléments sont particulièrement à retenir. Les carrières des gardiens de la paix ne sont pas, comme celles des préfets et hauts fonctionnaires de la PP, liées à un territoire incluant l'empire colonial. Cette remarque a un caractère d'évidence si l'on garde à l'esprit que la police parisienne est alors une administration territoriale ${ }^{58}$. De ce fait notamment, le capital colonial des gardiens parisiens est quasi complètement confondu avec leurs expériences militaires. Toute tentative d'expliquer certaines attitudes, comportements, pratiques

\footnotetext{
${ }^{56}$ Rapport de Maurice Legay directeur du SCAA, décembre 1961, APP H1 B30.

${ }^{57}$ Les difficultés de recrutement à la fin des années 1950 étaient telles que le "concours" d'entrée fut plus que jamais une simple formalité, facilitée pour les appelés en Algérie par le fait que des sessions d'examen de la PP y furent organisées.

${ }^{58}$ La différence est d'importance par rapport à la situation des policiers de la Sûreté nationale : les CRS couvraient l'ensemble des départements français et faisaient des missions régulières en Algérie bien avant la guerre (qui multiplie ces rotations malgré la création de compagnies basées dans les départements algériens). Même si les cadres des polices du Maghreb et de métropole étaient distincts, il était possible par permutation de poste de passer de l'un à l'autre.
} 
professionnels de par le passage par les colonies est ainsi rendu complexe par l'impossibilité de démêler ce qui relève de la socialisation militaire et de l'expérience coloniale.

Cette analyse rapide des trajectoires, entre métropole et colonies, des gardiens de la paix parisiens montre clairement que la PP ne dispose pas en interne d'importantes compétences coloniales : si les préfets de police sont incontestablement insérés dans des trajectoires et réseaux professionnels connectés à l'espace colonial, il semble que ce soit peu le cas des autres personnels. La minorité de gardiens de la paix ayant accumulé un certain capital colonial l'a fait au sein de l'institution militaire au travers de courts séjours Outre-mer (quelques mois le plus souvent, très rarement plus d'un an). Sans même évoquer les compétences linguistiques, cela apparaît très nettement insuffisant pour développer la connaissance, par exemple, des «milieux nord-africains », si souvent invoquée comme une nécessité préalable afin d'exercer la police des populations colonisées émigrées en région parisienne. Ainsi, il semble bien que, quand fut recréée, en 1953, une section de la police judiciaire (la brigade des agressions et violences, BAV) spécialisée dans la répression et le contrôle des Algériens de Paris ${ }^{59}$, cette rareté des compétences coloniales se fit cruellement ressentir. Alors que la BAV était censée représenter la pointe avancée d'une police de voie publique dynamique et moderne, d'anciens inspecteurs de la BNA y furent reversés ${ }^{60}$. Surtout, tous ceux ayant exercé en Algérie semblent y avoir été affectés d'office, quand bien même ils n'en avaient pas exprimé le désir et n'avaient qu'une connaissance très approximative de la langue arabe ${ }^{61}$. Les inspecteurs ayant une expérience coloniale quelconque (notamment de par un engagement dans la Marine) furent fortement encouragés à la rejoindre ${ }^{62}$. Les rares Français musulmans d'Algérie y ayant été recrutés n'avaient pas fait l'objet d'une sélection suffisante pour que certains d'entre-eux ne jouent pas double jeu avec les nationalistes du $\mathrm{FLN}^{63}$. Malgré ces incitations et la faible sélection à l'entrée, l'objectif initial d'avoir une brigade constituée au moins pour moitié de locuteurs kabyles ou arabes, ou a minima de policiers maîtrisant ces langues, ne fut ainsi jamais atteint. D'une certaine façon, la BAV, même si elle était véritablement spécialisée sur la seule clientèle algérienne, et plus encore la

\footnotetext{
${ }^{59}$ E. Blanchard, «Police judiciaire et pratiques d'exception pendant la guerre d'Algérie », Vingtième siècle. Revue d'histoire, n 90, 2006, p. 61-72.

${ }^{60}$ D'après un état des effectifs de janvier $1958(\mathrm{~N}=123)$, nous avons relevé qu'au moins quatre des policiers de la BAV étaient des anciens de la BNA. APP HA 89.

${ }^{61}$ Entretien avec M. Joseph Simon, Gosné (35), 20 juillet 2004.

${ }^{62}$ Récit de vie de M. Maurice Gouny.

${ }^{63}$ En 1958, il y avait entre deux et quatre « Français musulmans d'Algérie » à la BAV (tous contractuels) : l'un d'eux renseignait le FLN et avait notamment transmis des fiches de renseignement sur ses collègues. Il réussit à prendre la fuite en Algérie où il serait, après l'indépendance, devenu commissaire divisionnaire. R. LE TAILlANTER, "Le Grand". Ma vie de flic, Paris, Le Grand livre du mois, 1995, p. 101 ; récit de vie de M. Maurice Gouny.
} 
criminalité liée aux partis nationalistes, ne fut jamais vraiment une police coloniale - au sens où elle se serait inspirée des modalités de fonctionnement ou des pratiques de services outremer -, ni même un service à fort capital colonial ${ }^{64}$.

Cette relative pénurie interne de compétences forgées en situation coloniale explique que, quand Maurice Papon plaça la lutte contre les nationalistes algériens au coeur de ses priorités (1958-1962), il se détourna des personnels et des compétences de la PP. Il fit alors appel à des officiers qui impulsèrent une militarisation inédite de la police parisienne.

La question de l'intégration des préfets de police à l'espace colonial est avérée tant est évidente la place de l'Outre-mer dans leurs carrières professionnelles. En revanche, celle de l'influence de ce capital colonial sur leurs décisions et politiques à la tête de la police parisienne est beaucoup plus délicate. Pour ne prendre que l'exemple de Maurice Papon, son extrême longévité à la tête de la PP s'explique aussi par le fait qu'il sut s'affranchir du soutien des défenseurs les plus marqués de l'Algérie française pour se convertir au programme gaulliste d'acceptation de l'indépendance. L'expérience et les soutiens coloniaux de Papon n'étaient donc qu'une des facettes de ses ressources en matière de contribution à la définition d'une politique d'encadrement et de répression des Algériens immigrés dans le département de la Seine.

Il convient aussi de noter que d'autres préfets de police, ayant le même entregent colonial mais pris dans d'autres configurations politiques, ont pu mener des politiques dont les fondements et mises en œuvre étaient fort différents de ceux de Maurice Papon : les prises de position d'André-Louis Dubois en donnent un exemple éclatant.

Le prestige des fonctions outre-mer a contribué à insérer les préfets de police dans des réseaux coloniaux. Il n'est cependant pas possible d'affirmer, au contraire de l'exemple anglais $^{65}$, que les expériences coloniales étaient un préalable ou un accélérateur des carrières des policiers français. Les policiers des corps d'outre-mer avaient les plus grandes difficultés à être réintégrés dans les échelles administratives métropolitaines et n'étaient pas sans susciter la méfiance de collègues dont ils risquaient de retarder l'avancement et qui n'hésitaient pas à mettre en cause leurs méthodes de travail.

\footnotetext{
${ }^{64}$ Les inspecteurs de la BAV étaient recrutés sans modalités dérogatoires par le biais des concours de la PJ. Sur les 24 dont les lieux de naissance ont pu être relevés, aucun n'est né outre-mer et les deux tiers sont originaires du département de la Seine. Sur ces points aussi, les inspecteurs de la BAV ne se singularisent pas des autres inspecteurs de la PJ.

${ }_{65}$ J. WHITFIELD, Unhappy dialogue. The Metropolitan Police and black Londoners in post-war Britain, Devon, Willan publishing, 2004, p. 38.
} 
Surtout, l'influence des continuités coloniales entre métropole et outre-mer doit être fortement relativisée si l'on se réfère à l'ensemble du personnel de la PP et non aux seuls préfets. Après que la PP a supprimé à la Libération ses services directement inspirés d'institutions coloniales $^{66}$ et aux règles de recrutement dérogatoires afin de faciliter l'embauche d'agents “passés par les colonies”, son personnel, en particulier à l'échelon des gardiens de la paix, n'avait qu'une expérience très réduite du monde et des populations coloniales. Cette absence de capital colonial en interne et les résistances observées quant au transfert en métropole d'un certain nombre de pratiques courantes en Algérie ${ }^{67}$, conduisirent d'ailleurs Maurice Papon à contourner les directions et mode de recrutement traditionnels de la PP pour constituer une avant garde militaro-policière dans la lutte contre les nationalistes algériens.

Cette relative extériorité de la police parisienne au monde colonial doit cependant être nuancée : si elle se vérifiait à la PP jusqu'à la fin des années 1950, cela changea par la suite. De plus, la situation était différente à la Sûreté nationale qui administrait certaine des villes de banlieue. Les permutations de personnel, la fusion des polices, puis les rapatriements des policiers du Maroc et de Tunisie y avaient constitué une solide minorité d'inspecteurs ayant pratiqué leur métier des deux côtés de la Méditerranée ${ }^{68}$. Cette socialisation aux combats coloniaux d'une part croissante des policiers parisiens ne fut sans doute pas sans renforcer certaines pratiques répandues de violences (passage à tabac, ratonnades $^{69}$ ).

Il ne s'agit pas de postuler a priori que l'augmentation de la proportion de policiers ayant connu une expérience coloniale a durablement influé les pratiques de la police parisienne dans les années 1960. Il est cependant patent que ces années de fort renouvellement d'effectifs de la PP, restés très stables depuis la Libération, ont correspondu au passage de témoin entre une génération "Seconde Guerre mondiale" et une génération "guerre d'Algérie". Cette transition sans rupture (tant sont nombreux ceux entrés au cours de la Seconde Guerre mondiale et en poste jusqu'aux années 1970) eut notamment pour caractéristique que c'est à l'heure des décolonisations que le souvenir et l'expérience des colonies devinrent une caractéristique partagée d'une part importante des effectifs de la Préfecture de police.

\footnotetext{
${ }^{66}$ Les services de la rue Lecomte étaient ainsi appelés «la commune-mixte de Paris » - du nom d'une circonscription administrative d'Algérie.

${ }^{67}$ E. Blanchard , op. cit. , 2006.

${ }^{68}$ L'analyse des dossiers personnels d'inspecteurs et de commissaires ayant exercé à Argenteuil entre 1958 et 1962, montre que cette minorité se renforce au fur et à mesure que le conflit algérien se prolonge. Ces expériences sont cependant fort diverses. Pour ne citer que ceux passés par l'Algérie, quoi de commun entre le policer pied-noir rapatrié, l'appelé des combats de la guerre d'indépendance algérienne, l'inspecteur ayant effectué une courte mission pour lutter contre l'OAS ou celui muté pour régénérer les effectifs d'une police algérienne aux pratiques critiquées par la hiérarchie de la SN.

${ }^{69}$ Nombre de témoins, dont le discours n'est pas par ailleurs celui d'une défense des droits des citoyens face à la répression policière, donnent des exemples de perpétuation de ces violences, en particulier vis à vis des immigrés d'Afrique du Nord, bien après le cessez-le-feu en Algérie.
} 
